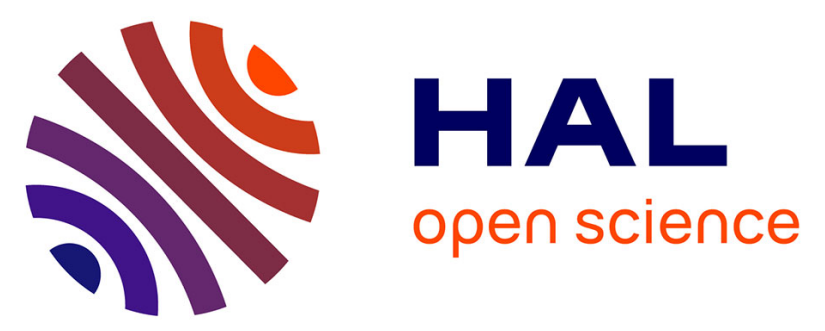

\title{
X-ray magnetic circular dichroism measured at the Fe K -edge with a reduced intrinsic broadening: x-ray absorption spectroscopy versus resonant inelastic $\mathrm{x}$-ray scattering measurements
}

Amélie Juhin, Philippe Sainctavit, Katharina Ollefs, Marcin Sikora, Adriano Filipponi, Pieter Glatzel, Fabrice Wilhelm, Andrei Rogalev

\section{To cite this version:}

Amélie Juhin, Philippe Sainctavit, Katharina Ollefs, Marcin Sikora, Adriano Filipponi, et al.. X-ray magnetic circular dichroism measured at the Fe K -edge with a reduced intrinsic broadening: x-ray absorption spectroscopy versus resonant inelastic x-ray scattering measurements. Journal of Physics: Condensed Matter, 2016, 28 (50), pp.505202 10.1088/0953-8984/28/50/505202 . hal-01421069

\section{HAL Id: hal-01421069 \\ https://hal.sorbonne-universite.fr/hal-01421069}

Submitted on 21 Dec 2016

HAL is a multi-disciplinary open access archive for the deposit and dissemination of scientific research documents, whether they are published or not. The documents may come from teaching and research institutions in France or abroad, or from public or private research centers.
L'archive ouverte pluridisciplinaire HAL, est destinée au dépôt et à la diffusion de documents scientifiques de niveau recherche, publiés ou non, émanant des établissements d'enseignement et de recherche français ou étrangers, des laboratoires publics ou privés. 


\title{
X-ray Magnetic Circular Dichroism measured at the Fe $K$-edge with a reduced intrinsic broadening: X-ray Absorption Spectroscopy vs Resonant Inelastic X-ray Scattering measurements
}

\author{
Amélie Juhin \\ E-mail: juhin@impmc.upmc.fr \\ Institut de Minéralogie, de Physique des Matériaux et de Cosmochimie (IMPMC), Sorbonne \\ Universités, UMR CNRS 7590, UPMC Univ Paris 06, Muséum National d'Histoire Naturelle, IRD \\ UMR206, 4 Place Jussieu, F-75005 Paris, France

\section{Philippe Sainctavit} \\ Institut de Minéralogie, de Physique des Matériaux et de Cosmochimie (IMPMC), Sorbonne \\ Universités, UMR CNRS 7590, UPMC Univ Paris 06, Muséum National d'Histoire Naturelle, IRD \\ UMR206, 4 Place Jussieu, F-75005 Paris, France
}

\section{Katharina Ollefs}

European Synchrotron Radiation Facility, BP 220, 38043 Grenoble Cedex, France

\section{Marcin Sikora}

Academic Centre for Materials and Nanotechnology, AGH University of Science and Technology, 30-059 Kraków, Poland

\section{Adriano Filipponi}

Dipartimento di Scienze Fisiche e Chimiche, Università degli Studi dell'Aquila, I-67100 L'Aquila, Italy.

\section{Pieter Glatzel}

European Synchrotron Radiation Facility, BP 220, 38043 Grenoble Cedex, France

\section{Fabrice Wilhelm}

European Synchrotron Radiation Facility, BP 220, 38043 Grenoble Cedex, France

\section{Andrei Rogalev}

European Synchrotron Radiation Facility, BP 220, 38043 Grenoble Cedex, France

\begin{abstract}
X-ray Magnetic Circular Dichroism is measured at the Fe $K$ pre-edge in Yttrium Iron Garnet using two different procedures that allow reducing the intrinsic broadening due to the $1 s$ corehole lifetime. First, deconvolution of XMCD data measured in Total Fluorescence Yield (TFY) with an extremely high signal-to-noise ratio enables to gain a factor of 2.4 in the XMCD intensity. Ligand Field Multiplet calculations performed with different values of intrinsic broadening show that deconvolving such high quality XMCD data is similar to reducing the lifetime broadening from a $1 s$ corehole to a $2 p$ corehole. Second, MCD is measured by Resonant Inelastic X-ray Scattering spectroscopy as a function of incident energy and emission energy. Selection of a fixed emission energy, instead of using the TFY, allows enhancing the MCD intensity up to a factor of $\sim 4.7$. However, this significantly changes the spectral shape of the XMCD signal, which cannot be interpreted any more as an absorption spectrum.
\end{abstract}


PACS numbers: 75.25.-j,71.70.Ch,75.47.Lx,78.70.En,78.70.Dm 
Following the first measurements in the eighties [1], X-ray Magnetic Circular Dichroism (XMCD) has rapidly become a popular spectroscopy to probe in an element selective way the magnetic properties of materials as diverse as molecular magnets, multilayers, magnetosomes, nanoparticles, etc [2]. At the $L_{2,3}$ edges of transition metal ions or at the $M_{4,5}$ edges of rare earth elements, the technique has met an immediate success because the XMCD signal has a large intensity (typically 10-100\% of the isotropic signal) and it benefits from the support of the powerful magneto-optical sum rules [3, 4]. At the $K$ pre-edge of $3 d$ ions, one can in principle probe the same empty $3 d$ states as at the $L_{2,3}$ edges, but using electric quadrupole transitions that are possibly superimposed on electric dipole transitions to the empty 4 pstates in non-centrosymmetric sites. However, the large development of the technique at the $K$-edges has been hampered by several difficulties: $(i)$ at the $K$-edge the broadening of the spectrum is obviously much larger than at the $L_{2,3}$ edges, which makes the interpretation more difficult, (ii) the XMCD effect is rather small (typically $0.1-1 \%$ of the isotropic signal) and often obscured by the main edge tail, which makes it difficult to isolate the pre-edge transitions from those into the continuum, (iii) the absence of spin-orbit coupling on the core-hole prevents obtaining information on the spin magnetic moment of the $3 d$ orbitals.

As expressed in the Fermi "Golden Rule", a XAS spectrum is a sum over all final states of discrete transitions occuring between an initial state and a final state. These transitions are broadened by $(i)$ the instrumental resolution, which can be modeled by a Gaussian function, and (ii) the broadening due to the finite lifetime of the excited state, which results in the convolution of discrete states by a Lorentzian function. From the Multiple Scattering analysis developed with complex potential as it is done by Rehr and collaborators with the Hedin-Lundqvist potential [5], it appears that the main damping factor close to the edge is the core-hole lifetime and not the inelastic scattering of the photoelectron. Inelastic scattering is the major cause of damping at slightly higher kinetic energy, when it is larger than the plasmon energy of the absorbing medium. The corehole lifetime $\tau_{\text {corehole }}$ is related to the Full Width at Half Maximum (FWHM) of the Lorentzian function by $\Gamma_{\mathrm{L}}=\hbar / \tau_{\text {corehole }}[6]$. For $3 d$ ions, typical FWHM values are 0.1-0.5 eV for $L_{2,3}$ edges, versus $1-2 \mathrm{eV}$ for $K$-edges [7].

In the nineties, significant technical progress in synchrotron radiation sources and beamlines has allowed recording experimental data with unprecedented high resolution and low noise level, thus reducing tremendously the broadening due to poor instrumental resolution. It thus became obvious that a significant part of spectral information could further be retrieved by removing the intrinsic Lorentzian broadening, or at least part of it. This was the driving force for developing (i) numerical deconvolution procedures, as well as (ii) novel experimental methods. Amongst the deconvolution methods, Loeffen et al. were the first to propose a procedure based on Wiener filtering allowing the removal of half of the $2 p$ corehole lifetime broadening in the $L_{3}$ edge XAS spectra of rare-earths [9]. They pointed out that the deconvolution is practically limited by the value of the experimental Signal-to-Noise Ratio (SNR) through the Shannon-Hartley theorem of information theory. Several deconvolution procedures were developed and successfully applied to sharpen the $K$-edge spectra of light elements $[10,11,12,13]$. Recently, the $K$-edge XAS spectra of Gd and Pr were deconvolved from two-third of the large $1 s$ corehole lifetime broadening using the algorithm developed by Filipponi [14, 15]. Nevertheless, such deconvolution procedures have seldom been applied to $K$-edge XAS spectra of $3 d$ elements (i.e., at the $\mathrm{Cr}$ and V $K$ edges in $\left(\mathrm{Cr}_{x} \mathrm{~V}_{1-x}\right)_{2} \mathrm{O}_{3}$, and at the $\mathrm{Zn}$ and $\mathrm{Cu} K$-edges in complexed xerogels $\left.[16,17,13]\right)$ but never to XMCD data.

Table 1. Different contributions to the spectral broadening in the XAS/XMCD and RIXS/RIXS-MCD experiments performed in this work. All values are given in eV and represent a FWHM. The different broadenings are defined as in Ref. [8].

\begin{tabular}{|c|c|c|c|c|}
\hline detection & $\begin{array}{l}\text { instrumental broa } \\
\text { incident energy } \\
\text { bandwith } E_{I N C}\end{array}$ & $\begin{array}{l}\text { dening (Gaussian) } \\
\text { emission energy } \\
\text { bandwith } E_{E M I}\end{array}$ & $\begin{array}{l}\text { lifetime broadening } \\
\quad \text { (Lorentzian) }\end{array}$ & $\begin{array}{l}\text { total (Voigt) } \\
\text { using Eqs. }(1-2) \\
\text { of Ref. }[8]\end{array}$ \\
\hline TFY (XAS) & 1.0 & $\overline{-}$ & $\Gamma_{1 s}=1.2$ & 1.8 \\
\hline "TFY-like" (RIXS) & 0.23 & - & $\Gamma_{1 s}=1.2$ & 1.3 \\
\hline CEE (RIXS) & 0.23 & 0.97 & $\Gamma_{1 s}=1.2, \Gamma_{2 p}=0.40$ & 0.93 \\
\hline
\end{tabular}


On the experimental side, measurement of XAS spectra with a reduced lifetime broadening was achieved thanks to the developement of spectrometers that make use of analyzer crystals for the detection. Under the impulsion of J. Hastings, following the groundbreaking work of Hamalainen et al. in 1991 [18], Resonant Inelastic X-ray Scattering spectroscopy has become a major high resolution technique based on a resonant photon-in, photon-out process $[19,20]$. However, it was soon realized that XAS spectra detected in such a way were not only significantly sharpened, but that in many cases they could differ dramatically in shape and intensity from standard XAS spectra [21, 9, 8]. Moreover their angular and polarization dependences are more complex than x-ray absorption. Their detailed interpretation thus often requires the support of calculations. Alternatively, for $1 s 2 p$ RIXS measurements (which probe the evolution of the $K \alpha$ emission $(2 p \rightarrow 1 s)$ following the resonant excitation of a $1 s$ electron), a numerical treatment of the Kramers-Heisenberg equation has been proposed to derive a lifetime-broadening-suppressed XANES spectrum [22].

Recently, $1 s 2 p$ RIXS was combined with magnetic circular dichroism (RIXS-MCD) in the hard x-ray range [23]. In the case of $3 d$ transition metal ions, it is typically studied over the $K$ pre-edge range, probing unoccupied states predominantly composed of the $3 d$ magnetic orbitals. It was stated that it could be a valuable alternative to soft x-ray XMCD, when dealing with materials that are challenging to investigate with the latter (i.e., with a buried or complex internal structure, such as multilayers or coreshell nanoparticles) or when using demanding sample environments such as liquids or high pressure cells $[24,25]$. In $3 d$ metal oxides, the RIXS-MCD signal at the pre-edge can be up to an order of magnitude more intense with respect to the edge jump than XMCD measured in Total Fluorescence Yield (TFY) detection. Both the reduction in lifetime broadening and the resonant, two-photon nature of the RIXS process are intimately related and result in this intensity enhancement. However, one should be careful not to interpret RIXS-MCD as a conventional XMCD spectrum: although this precaution may be trivial, RIXS is often readily interpreted in the literature using arguments based on the absorption cross-section rather than the Kramers-Heisenberg expression.

In the present paper, we examine the possibility to enhance the XMCD intensity at the $K$ pre-edge of a $3 d$ ion by applying two different approaches. The case of Fe in Yttrium Iron Garnet (YIG) was chosen as benchmark because the XMCD signal at the Fe $K$ pre-edge has been previously measured and interpreted [26], and it has a derivative-like, simple shape. On one side, we apply a deconvolution procedure to XMCD data measured in TFY detection with an excellent SNR, with the goal to reduce the effect of the finite corehole lifetime. On the other side, RIXS-MCD data were measured on similar samples with a spectrometer. Comparison of the spectral shape and intensity in both kinds of data, supported by Ligand Field Multiplet calculations, provide a clear understanding of what can be gained from the reduction in intrinsic broadening and from the RIXS detection scheme, respectively. It is shown that, (i) TFY-XMCD at the $K$-edge of transition metals is proportional to a "true" absorption measurement, in which all spectral features can be enhanced by reduction of lifetime broadening, (ii) RIXS-MCD in the pre-edge is not an absorption measurement, but additional information can be extracted by changing the emission energy. This combined study thus clarifies the complementarity between $K$-edge XMCD and $1 s 2 p$ RIXS-MCD spectroscopies when investigating the magnetic properties of materials.

\section{Methods}

\section{1. $X A S$ and $X M C D$ experiments}

XAS and XMCD spectra were collected at the Fe $K$-edge in the fluorescence mode at ID12 beamline of the ESRF (Grenoble, France), using a two-crystal monochromator equipped with a pair of Si(111) yielding an energy resolution $\Delta E=1.04(1) \mathrm{eV}$ at the Fe $K$-edge with the fundamental harmonic of a helical undulator. The circular polarization is delivered by an Apple-II helical undulator with a polarization rate $P_{c}$ of $\sim 93 \%$ at the Fe $K$-edge. The measurements were performed at room temperature in an applied magnetic field of $\pm 0.9 \mathrm{~T}$, with an angle between the X-ray beam and the sample normal equal to $45^{\circ}$. XAS spectra were measured by switching alternatively the circular polarization and the direction of magnetic field. In total, seventy-six spectra ranging from $7100 \mathrm{eV}$ to $7172 \mathrm{eV}$ were recorded with an energy step of $0.04 \mathrm{eV}$ and a counting time of one second per point. The sample is a thin orientated film of Yttrium Iron Garnet grown by liquid phase epitaxy on a Gadolinium Gallium Garnet substrate [26]. The film has a thickness of $9.8 \mu \mathrm{m}$ and the normal surface aligned with the (111) axis of the garnet 
XMCD with reduced intrinsic broadening

cubic cell. XMCD spectra were normalized to perfect circular polarization degree (i.e., divided by $P_{c}$ ), but they were not corrected for self-absorption effects which could be neglected in the pre-edge region for this thin film sample. The "isotropic" (polarization averaged) XAS spectrum is obtained by averaging two XAS spectra measured with opposite photon helicities and identical magnetic field, while the XMCD spectrum is obtained from their difference. The absorption edge jump is set to 1 for all XAS spectra.

\subsection{RIXS and RIXS-MCD experiments}

RIXS and RIXS-MCD measurements were performed at ID26 beamline of the ESRF, using incoming radiation from the undulator fundamental monochromatized with a pair of $\operatorname{Si}(311)$ crystals. The circular polarization was generated from the horizontally polarized beam using a $500 \mu \mathrm{m}$ thick diamond (111) quarter wave plate (QWP). The inelastically scattered photons were analyzed using a vertical spectrometer composed of four spherically bent Ge(440) Bragg crystals in the Rowland geometry $(R \sim 1 \mathrm{~m})$ and detected by an Avalanche Photo Diode. The combined resolution of the setup was determined as full width at half maximum of elastic scattering was $\Delta E=1.0(1) \mathrm{eV}$. The $1 s 2 p$ RIXSMCD spectra were measured at room temperature as a set of Constant Emission Energy (CEE) scans over the $K \alpha_{1}$ and $K \alpha_{2}$ fluorescence lines. A RIXS plane is thus a $2 D$ representation of the fluorescence intensity as a function of incident energy and emission energy. Spectra were recorded in the range $7110 \mathrm{eV}-7122 \mathrm{eV}$ for the incident energy (by step of $0.15 \mathrm{eV}$ ) and $6387 \mathrm{eV}-6408 \mathrm{eV}$ for the emission energy (by step of $0.2 \mathrm{eV}$ ), with an acquisition time of one second per energy point. The sample was kept in magnetic saturation using a $\mathrm{Nd}_{2} \mathrm{Fe}_{14} \mathrm{~B}$ permanent magnet ( $\mathrm{B} \sim 0.3 \mathrm{~T}$ on the sample surface), while the photon helicity was reversed after two CEE spectra. The constant degree of circular polarization, $P_{c} \sim 75(5) \%$, was maintained by simultaneous scanning the incident photon energy and $\theta_{\mathrm{QWP}}$. The sample is a thin orientated film of YIG with a thickness of $6.0 \mu \mathrm{m}$, grown on a Gadolinium Gallium Garnet substrate. The "isotropic" RIXS plane is obtained by averaging the RIXS planes obtained with left and right circular polarization, while the RIXS-MCD plane is obtained from their difference. The RIXS planes measured with polarized light were normalized such that the maximum of isotropic RIXS plane equals unity (i.e., taking the maximum at $7115 \mathrm{eV}$ incident energy, $6404.7 \mathrm{eV}$ emission energy). Note that RIXS and RIXS-MCD data are normalized to perfect circular polarization rate, but not corrected for selfabsorption effects, which are assumed to be negligible in the pre-edge range. From SQUID magnetization measurements, the YIG sample is fully magnetized when magnetic induction is larger than $250 \mathrm{mT}$ [27]. Hence the experimental conditions that were used on both ID26 (0.3T) and ID12 (0.9T) beamlines ensure a completely saturated sample.

\subsection{Deconvolution of $X A S$ and $X M C D$ spectra}

In order to deconvolve the XAS and XMCD $K$-edge spectra, we have used the deconvolution procedure developed by Filipponi and implemented in the DECONV program of the GNXAS package [28, 29]. In this program, the deconvolution of a Lorentzian function is performed by first Fourier Transforming the absorption spectrum and then applying the deconvolution itself in Fourier space with a window function. The deconvolved function is then back transformed to direct space. However, the full deconvolution of the core-hole width $\Gamma_{c}$ (FWHM) cannot be achieved due to noise blow-up effect. To avoid this, a Gaussian filter with FWHM equal to $\sigma$ is applied, where $\sigma$ is chosen at best in the range $\Gamma_{c} / 3<\sigma<\Gamma_{c} / 2$. The value of $\sigma$ is chosen as low as possible (although avoiding noise blow up), and the optimized value is dependent on the Signal-to-Noise Ratio of the original experimental (the higher the SNR, the lower the value of $\sigma$ ). All details of this procedure can be found in Ref. [15]. The deconvolved XMCD and "isotropic" XAS spectra were obtained from direct deconvolution of the experimental XMCD and "isotropic" XAS data, respectively.

\subsection{Ligand Field Multiplet calculations}

The crystal field multiplet approach developed by Thole and Cowan was used to simulate XAS and XMCD spectra at the Fe $K$ pre-edge [30,31]. This approach takes into account all the multielectronic Coulomb interactions ( $3 d-3 d$ repulsions, and also $1 s-3 d$ repulsions in the XAS final state), as well as the spin-orbit coupling on every open shell of the absorbing atom, and it treats its geometrical environment through a 
XMCD with reduced intrinsic broadening

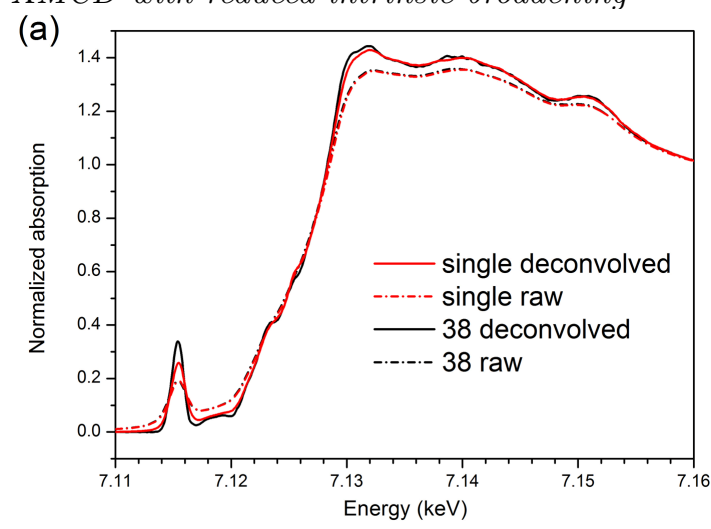

(b)

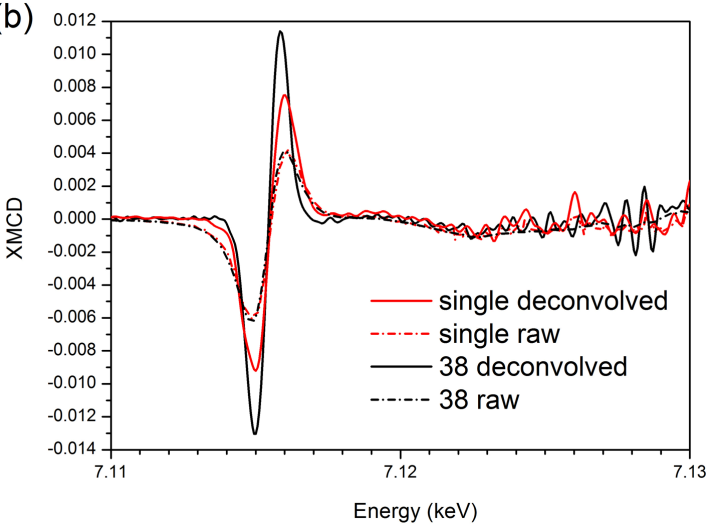

Figure 1. Comparison between raw XAS and XMCD data (dashed lines) and deconvolved spectra (solid lines) obtained at the Fe $K$-edge in the YIG thin film with $9.8 \mu \mathrm{m}$ thickness. Data obtained from one single pair of spectra measured with opposite helicities are shown in red, while those obtained from the average of 38 pairs (19 pairs were measured for one direction of the field, and other 19 for the opposite direction) are plotted in black.

crystal-field potential. Calculations were performed for a $\mathrm{Fe}^{3+}$ ion located in the tetrahedral site of the YIG structure $\left(T_{d}\right.$ point group symmetry) and for a $\mathrm{Fe}^{3+}$ ion located in the octahedral site (taking $O_{h}$ point group symmetry and neglecting the small trigonal distortion). We have started from the pioneering work of Arrio et al. [32], who first performed $K$ pre-edge XAS calculations in non-centrosymmetric sites, and we have extended it to the calculation of XMCD. The isotropic XAS and XMCD cross-sections were calculated using the Fermi Golden Rule. For the $O_{h}$ site, since the inversion symmetry prevents the existence of $3 d-4 p$ mixing on the Fe site of the same atom, the pre-edge intensity arises from electric quadrupole transitions solely and it is described by the transitions from the initial state $1 s^{2} 3 d^{5}$ to the final state $1 s^{1} 3 d^{6}$. For the $T_{d}$ site, the cross-section is calculated as the sum of electric dipole and electric quadrupole transitions using the non-relativistic expression given in Ref.15 of Arrio et al. [32] The initial state and the final state are each described by a mixture of two configurations, respectively $1 s^{2} 3 d^{5}+1 s^{2} 3 d^{4} 4 p^{1}$ and $1 s^{1} 3 d^{6}+1 s^{1} 3 d^{5} 4 p^{1}$, using a hybridization Hamiltonian whose mixing intensity $V_{p d}$ was taken equal to that of Ref. [32]. The crystal field parameter $10 D q$ was taken as $0.7 \mathrm{eV}$ for the $T_{d}$ site and $1.5 \mathrm{eV}$ for the $O_{h}$ site [32]. The external magnetic field was set parallel to the 4 -fold axis of the tetrahedral (octahedral) site and we used an exchange field corresponding to a Zeeman splitting of $10 \mathrm{meV}$. The radial integrals for interelectronic repulsion and spin-orbit coupling were calculated using the atomic Hartree-Fock code of Cowan, where the Slater integrals were scaled down to $80 \%$. We underline the fact that absolute absorption cross-sections have been calculated in $\AA^{2}$, for the electric dipole part on one hand and the electric quadrupole part on the other hand (both at $\mathrm{T}=300 \mathrm{~K}$ ). The population of the initial state levels at a finite temperature was determined by a Boltzmann law. The transition lines were convoluted using a Lorentzian function and a Gaussian function to account for the core-hole lifetime and instrumental resolution, respectively. The FWHM of the Gaussian was chosen a $1.0 \mathrm{eV}$ in agreement with the theoretical value for a $\mathrm{Si}(111)$ double crystal monochromator at $7.1 \mathrm{keV}$. For the Lorentzian, the FWHM was first chosen as $1.25 \mathrm{eV}$, which is the tabulated value for the Fe $1 s$ core hole from Ref. [7]. In a second calculation, a reduced Lorentzian broadening of $0.4 \mathrm{eV}$ (FWHM) was chosen to mimic the effect of apparent reduced lifetime broadening that is obtained when extracting Constant Emission Energy line scans from RIXS planes. This value is close to the Lorentzian broadening of the Fe $2 p$ hole that is present in the RIXS final state. Finally, the transitions are normalized to the edge jump at the Fe $K$-edge, calculated for an Fe atom from Ref. [33] as $3.310^{-4} \AA^{2}$. Hence, the calculated XAS and XMCD spectra can be directly compared to the normalized experimental ones. 
Normalized RIXS



Normalized RIXS-MCD

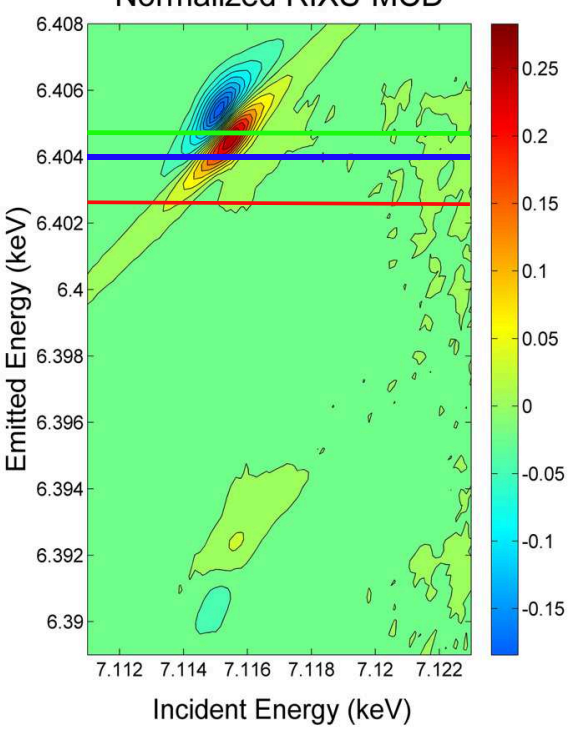

Figure 2. $1 s 2 p$ RIXS plane (left) and RIXS-MCD plane (right) measured at the Fe $K$-edge in the YIG thin film with $6.0 \mu \mathrm{m}$ thickness. Both planes are normalized to the maximum of the RIXS intensity measured in the pre-edge region (at $7115 \mathrm{eV}$ incident energy, $6404.7 \mathrm{eV}$ emission energy). Colored horizontal lines correspond to the CEE scans plotted in Figure 3.

\section{Results and discussion}

\subsection{Deconvolution of $X M C D$ data}

Figure 1 shows the high SNR experimental XAS and XMCD spectra measured at the Fe $K$-edge in the YIG thin film together with the corresponding deconvolved XAS and XMCD spectra. For the raw data obtained from a single pair of XAS spectra (dashed red lines), the XMCD signal in the pre-edge region has a peak-to-peak intensity of $\sim 1.10^{-2}$ with respect to the edge jump, while the maximum of the pre-edge intensity is 0.19 . This is in agreement with the data previsouly measured on this very sample and other Fe-bearing garnets [26]. In Figure 1 are also plotted the deconvolved XMCD and polarization averaged XAS spectra obtained from the deconvolution procedure. For such a noise level of approximately $\sim 2.10^{-4}$, the deconvolution of the data has been maximized using a Lorentzian with FWHM of $1.25 \mathrm{eV}$ and a Gaussian filter with FWHM of $0.84 \mathrm{eV}$ (i.e., the Gaussian FWHM cannot be further decreased without introducing noise blow up). Both the deconvolved XMCD signal and the XAS spectrum show spectral features that are sharper and more intense than in the original data: in the pre-edge region, the peak-to-peak intensity of the XMCD signal is multiplied by 1.7, while the maximum of the XAS is enhanced by a factor 1.36. For the data averaged from 38 pairs of spectra (black lines), the noise level is reduced to $\sim 6.10^{-5}$ which enables to push the deconvolution further, by using $0.46 \mathrm{eV}$ for the FWHM of the Gaussian filter. The resulting deconvolved XMCD spectrum shows a strong peak-to-peak intensity (0.024) in the pre-edge range, which represents an enhancement factor of 2.4 with respect to the original (non-deconvolved) data. Meantime, the maximum of the XAS pre-edge intensity is increased by a factor 1.77 .

\subsection{Photon-in, photon-out XMCD signal measured by RIXS}

Figure 2 shows the experimental $1 s 2 p$ RIXS and RIXS-MCD planes measured on the $6.0 \mu \mathrm{m}$ YIG thin film. The latter shows two groups of dichroic features located respectively at the $K \alpha_{1}$ and $K \alpha_{2}$ emission lines, that are each composed of two features with opposite sign. This spectral signature is very similar to the RIXS-MCD planes measured in other $\mathrm{Fe}^{3+}$-bearing samples $[23,25]$. Normalizing both planes such that the maximum of the isotropic RIXS plane in the pre-edge range (i.e., at $7114.9 \mathrm{eV}$ in incident energy and $6404.7 \mathrm{eV}$ in emission energy) is 1, the intensity of the RIXS-MCD effect is estimated to $48 \%$ 
XMCD with redused intrinsir hrondenina

(a)

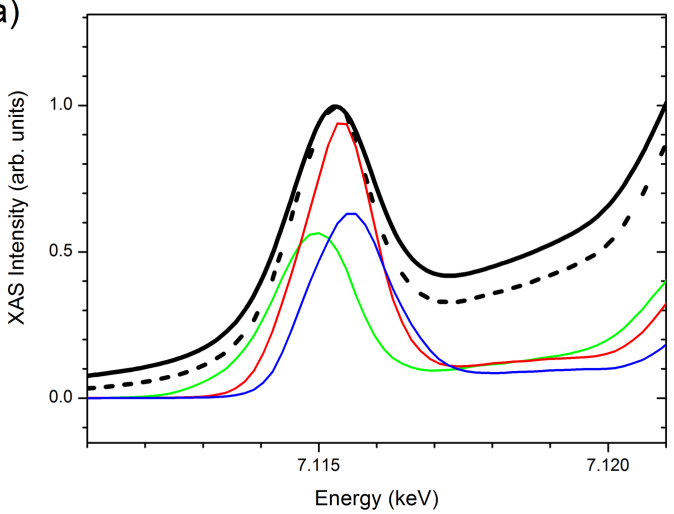

(b)

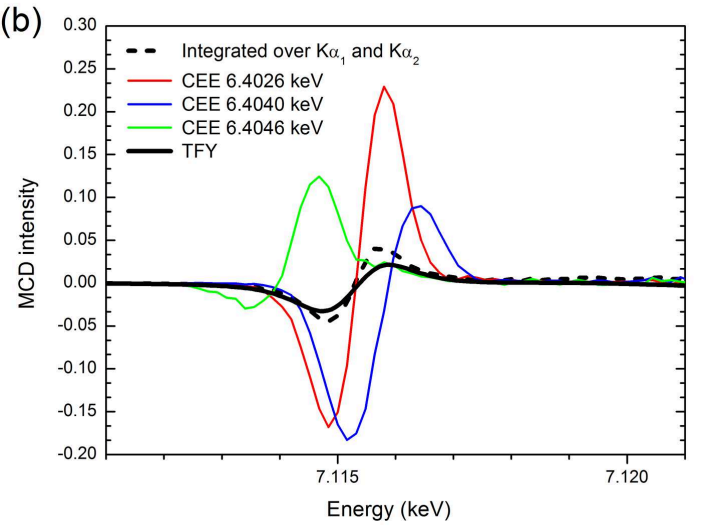

Figure 3. (a) CEE-XAS and (b) CEE-MCD $1 D$ plots extracted respectively from the isotropic RIXS and RIXS-MCD planes shown in Figure 2: Constant Emission Energy scans at $6402.6 \mathrm{eV}$ (green), $6404.0 \mathrm{eV}$ (red), $6404.6 \mathrm{eV}$ (blue), "TFY-like" data (black dashed) obtained from the integration of the plane along the emission energy direction, TFY (non-deconvolved) data from Figure 1 (black solid line). Note that in this figure the "TFY-like" and TFY data are normalized such that the maximum of the $\mathrm{XAS}$ in the pre-edge equals unity.

peak-to-peak. This is fully compatible with a dominant contribution from the tetrahedral $\mathrm{Fe}^{3+}$ ions of the garnet structure. Indeed the RIXS-MCD intensity is expected to be only a few $\%$ of the total pre-edge intensity for the $\mathrm{Fe}^{3+}$ ions lying in the centrosymmetric octahedral sites $\left(C_{3 i}\right)$ of the garnet structure, due to the absence of allowed $p-d$ mixing which prevents the occurence of intense electric dipole transitions in the absorption step of the RIXS process. Similarly, the dominant dichroic contribution to the RIXS-MCD plane of magnetite was interpreted as arising from the tetrahedral $\mathrm{Fe}^{3+}$ ions, with an intensity reaching $\sim 30 \%$ (using the same normalization as in the present work) [23].

One-dimensional spectra were then extracted from the normalized RIXS and RIXS-MCD planes. They are shown in Figure 3 as CEE scans that correspond to horizontal cuts of the plane, by setting the spectrometer energy for example to $6402.6 \mathrm{eV}$ (green), $6404 \mathrm{eV}$ (red) or $6404.6 \mathrm{eV}$ (blue). They are compared to the XAS and XMCD spectra measured in TFY detection (data from Figure 1, black solid lines) and to those obtained from the integration of the RIXS and RIXS-MCD planes, respectively, over the whole $K \alpha_{1}$ and $K \alpha_{2}$ range ("TFY-like", black dotted lines). In CEE scans, the spectral broadening is significantly smaller than in the "TFY-like" spectra. In first approximation, one considers that it results from the reduction of lifetime broadening from $\Gamma_{1 s}$ to $\Gamma_{\mathrm{APP}}$ where [8]:

$$
\Gamma_{\mathrm{APP}} \sim \frac{\Gamma_{1 s} \Gamma_{2 p}}{\sqrt{\left(\Gamma_{1 s}\right)^{2}+\left(\Gamma_{2 p}\right)^{2}}}
$$

In the present case $\Gamma_{\mathrm{APP}}$ is close to the $2 p$ corehole lifetime broadening $(0.4 \mathrm{eV})$. Nevertheless, a more accurate determination of the spectral broadening in CEE scans shall include as well the broadening from the incident energy $E_{I N C}$ and from the emitted instrumental energy resolution $E_{E M I}$ (which is dependent on the spectrometer), as discussed in Ref. [8]. The spectral broadening was estimated to $0.93 \mathrm{eV}$ (FWHM), which is lower than for the "TFY-like" data (1.3 eV FWHM).

From Figures 2 and 3, it is clear that both the spectral shape and relative intensity of the features in the CEE-XAS and CEE-MCD spectr depend on the emission energy. The largest peak-to-peak intensity $(\sim 40.0 \%)$ in CEE-MCD is obtained for an emission energy of $6404.0 \mathrm{eV}$ (red line), with a signal showing first a negative intensity at $7115 \mathrm{eV}$ and then a positive peak at $7116 \mathrm{eV}$. When the emission energy is set to $6404.6 \mathrm{eV}$, the negative peak in the CEE-MCD scan (blue line) becomes more intense than the positive one, and both are shifted to higher incident energy values. The peak-to-peak CEE-MCD intensity is now $27.0 \%$. On the contrary, when the emission energy is set to $6402.6 \mathrm{eV}$, the negative peak in the CEE-MCD scan (green line) becomes less intense than the positive one, with a peak-to-peak CEE-MCD intensity of $15 \%$, and both peaks are shifted to lower incident energy values. This behaviour is intimately related to the bidimensional photon-in, photon-out character of the RIXS-MCD spectroscopy, which naturally brings complementary insight to the one-dimensional photon-in representation given by TFY detected 
XMCD with reduced intrinsic broadening

XMCD. The latter can be understood as an "average" image of the effect, which is therefore considerably less intense ( $8.4 \%$ peak-to-peak for the "TFY-like" spectrum) than, for example, the CEE-MCD signal measured with an emission energy of $6404.6 \mathrm{eV}$ (40.0\%). For the present data, the maximal enhancement factor that can be gained in MCD intensity by comparison to "TFY-like" data is roughly 4.7. It is due to (i) the smaller effective corehole lifetime in CEE-MCD scans as compared to TFY-MCD, and (ii) the existence of multiplet splittings in the RIXS final state, which enables to probe different final states depending on the emission energy chosen, and thus allows to maximize the effect by tuning the latter to the most dichroic features [9]. TFY-MCD can be related to the ground state properties while CEE-MCD also involves an excited state, which explains why its spectral shape has such a strong dependence on the emission energy. The possibility to enhance significantly the MCD intensity by using CEE rather than TFY detection is interesting for scientific cases where one wants to monitor the MCD signal as a function of an external parameter (magnetic field, temperature, pressure, time...). Nevertheless, one shall keep in mind that interpreting the shape and intensity of CEE-MCD scans without the support of the full RIXS-MCD plane and / or calculations is not possible.

Although the $K \alpha$ emission represents $\sim 90 \%$ of the fluorescence yield, the "TFY-like" XMCD spectrum obtained from the integration of the RIXS-MCD plane is $\sim 1.6$ more intense than the MCD signal measured directly using TFY detection. This difference can be mainly attributed to the instrumental energy broadening that is smaller in the "TFY-like" data due to the use of a monochromator with a higher resolving power (see Figure 3a and Table 1). In addition, part of this difference can result from the angular dependence of electric quadrupole transitions, which is known to be sizeable in the pre-edge region even for cubic crystals [34]. Indeed, single crystals of YIG with same nominal composition but different crystalline orientations were measured in both experiments, which may explain the measured discrepancy. This is also supported by the fact that an additional feature is observed at $7120 \mathrm{eV}$ on the deconvolved TFY-MCD spectrum (Figure 1), while it is absent in the data measured by RIXS.

\subsection{Analysis of the XMCD pre-edge features}

In order to quantify the proportion in the RIXS-MCD intensity enhancement that is due to the smaller lifetime broadening and the proportion that is due to the final state effects, we have performed Ligand Field Multiplet calculations. Figure 4 shows the theoretical XAS and XMCD pre-edge spectra calculated for a tetrahedral $\mathrm{Fe}^{3+}$ ion and an octahedral $\mathrm{Fe}^{3+}$ ion in the garnet structure, using a Gaussian energy broadening of $1.0 \mathrm{eV}(\mathrm{FWHM})$ and the Lorentzian lifetime broadening of the $1 s$ core hole $(1.25 \mathrm{eV}$ FWHM). Note that all XAS spectra are normalized to the edge jump, which allows direct comparison to experimental data. Table 2 summarizes for the different spectra the values of the absorption intensity at the XAS pre-edge maximum and for the peak-to-peak XMCD.

For the tetrahedral site, the isotropic XAS spectrum shows one main feature whose intensity reaches $11 \%$ of the edge jump, which is in agreement with previous experimental and theoretical work [32, 35]. It is dominated by electric dipole (E1) transitions that are twice more intense than electric quadrupole (E2) transitions $(7.4 \%$ vs $3.6 \%$ ). The XMCD signal has a symmetric, derivative shape and a peak-to-peak intensity of $0.69 \%$, which yields a XMCD / XAS ratio of 0.063 . Note that the relative proportion of E2 transitions with respect to E1 transitions is higher in the XMCD signal than in the isotropic spectrum, which reflects the fact that $3 d$ states are more magnetic than $4 p$ states. For the octahedral site, the pre-edge XAS is composed of two features with a maximum intensity of $2.7 \%$, consistently with the absence of $\mathrm{E} 1$ transitions due to the lack of local $p-d$ mixing. This is also consistent with $K$ pre-edge XAS data measured in other $3 d$ oxides $[32,34,36,35]$. The XMCD signal has a derivative shape with a peak-to-peak intensity of $0.3 \%$, yielding a XMCD / XAS ratio of 0.11 that is higher than for the tetrahedral site.

The XAS and XMCD spectra calculated with a smaller Lorentzian broadening (0.4 eV FWHM) are shown in the bottom panels of Figure 4. For the tetrahedral site, the maximum of the XAS spectrum reaches $18.8 \%$, i.e. a factor 1.7 more than with the larger lifetime broadening, while the XMCD signal is multiplied by 2.6 with a peak-to-peak intensity of $1.82 \%$. For the octahedral site, the XMCD intensity is multiplied by 2.6 (reaching $0.79 \%$ ), while the XAS intensity is enhanced by a factor 1.6. Our calculations show that for both types of $\mathrm{Fe}^{3+}$ ions, the reduction of Lorentzian broadening from the full $1 s$ corehole lifetime to the $2 p$ corehole lifetime allows enhancing the XMCD signal by a factor $\sim 2.6$ and the XAS signal by $\sim 1.5-1.7$. This gain in intensity is similar to what was obtained in Figure 1 by deconvoluting 
XMCD with redused intrinsir hrondenina

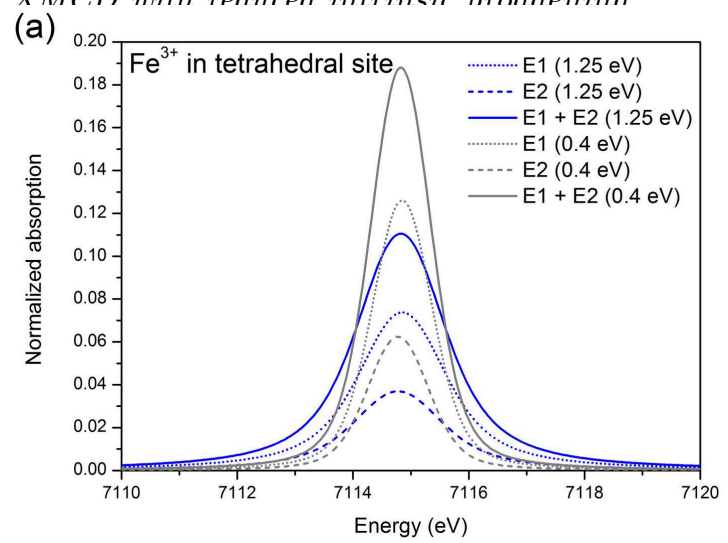

(c)

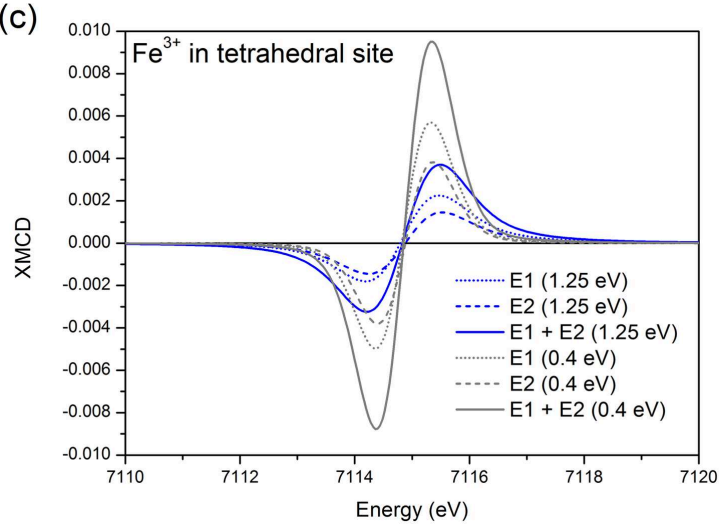

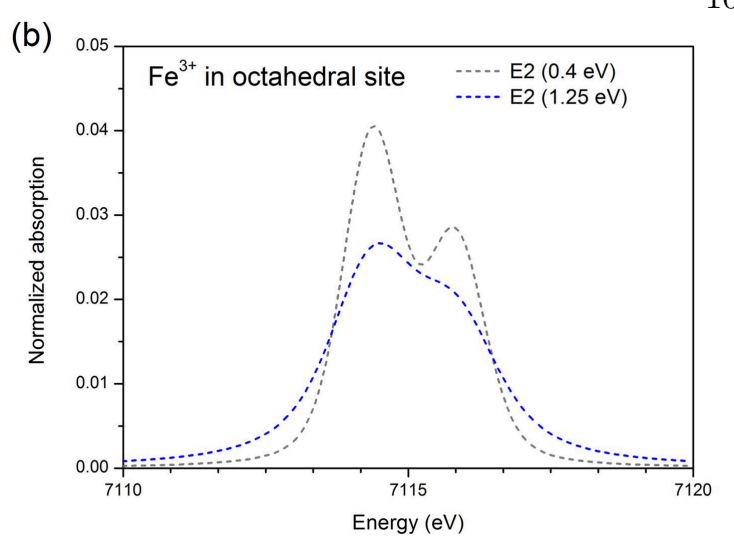

(d)

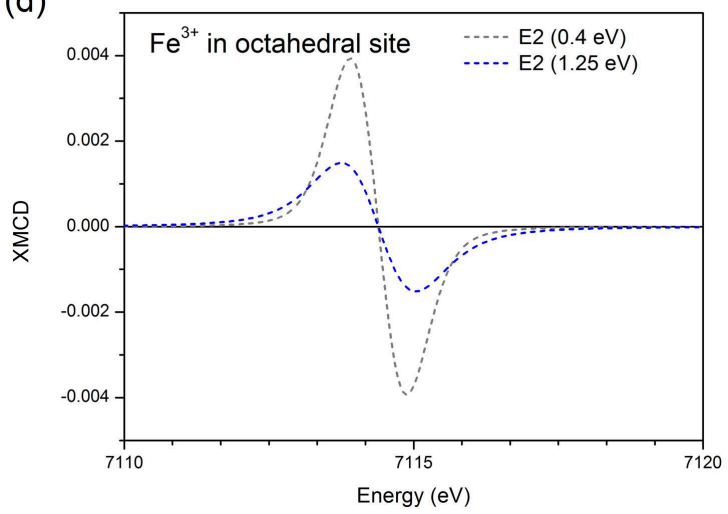

Figure 4. Theoretical isotropic XAS and XMCD spectra calculated in Ligand Field Multiplet theory for a $\mathrm{Fe}^{3+}$ ion in tetrahedral site (panels a and c) and octahedral site (panels b and d), using two different Lorentzian broadenings with FWHM equal to $1.25 \mathrm{eV}$ (solid) and $0.4 \mathrm{eV}$.

Table 2. Intensity of the isotropic XAS spectrum (taken at the maximum of the pre-edge) and of the XMCD spectrum (taken as the peak-to-peak intensity) in the experimental and theoretical spectra plotted in Figures 1 and 4. Intensities are given for an edge jump of 1.

\begin{tabular}{|c|c|c|c|c|c|c|c|}
\hline & \multicolumn{3}{|c|}{ TFY (exp) } & \multicolumn{3}{c|}{ TFY (calc) } \\
& raw & $\begin{array}{c}\text { single } \\
\text { deconvolved }\end{array}$ & $\begin{array}{c}38 \\
\text { deconvolved }\end{array}$ & $\begin{array}{c}T_{d} \text { site } \\
\Gamma_{\mathrm{L}}=1.25 \mathrm{eV}\end{array}$ & $\Gamma_{\mathrm{L}}=0.4 \mathrm{eV}$ & $\Gamma_{\mathrm{L}}=1.25 \mathrm{eV}$ & $\Gamma_{\mathrm{L}}=0.4 \mathrm{eV}$ \\
\hline Max. of Iso & 0.19 & 0.26 & 0.34 & 0.11 & 0.19 & 0.027 & 0.041 \\
\hline $\begin{array}{c}\text { XMCD } \\
\text { (peak-to-peak) }\end{array}$ & 0.010 & 0.017 & 0.024 & 0.0070 & 0.0018 & 0.0030 \\
\hline
\end{tabular}

the data measured in TFY with the highest SNR (average of 38 pairs) from two-third of the $1 s$ corehole lifetime broadening. We thus conclude that the large MCD intensity that can be measured in RIXS (up to $40 \%$ in CEE-MCD scans) arises only partly from the reduction of lifetime broadening. Most of the MCD enhancement is due to the existence of multiplet splittings in the RIXS final state, which are, in the present case, mainly the $2 p-3 d$ Coulomb repulsions. The choice of one emission energy selects a particular final state with a given MCD spectral shape, which may be different from that of another final state. Therefore, XMCD detected by RIXS shall not be used blindly and not be seen as a simple way of measuring XMCD spectra with an increased intensity and a reduced lifetime broadening.

\section{Conclusion}

We have compared the shape and intensity of the XMCD signal measured at the Fe $K$ pre-edge in YIG by two approaches allowing to reduce the intrinsic Lorentzian broadening, i.e., deconvolution of low noise 

one photon-out). A factor 2.4 is gained in the XMCD intensity by deconvolving XMCD spectra recorded with a noise level of $6 \cdot 10^{-5}$ from about two third of the $1 s$ corehole lifetime broadening. This result is quantitatively confirmed by Ligand Field Multiplet calculations. The XMCD intensity can be further enhanced by measuring Constant Emission Energy MCD scans close to the $K \alpha_{1}$ emission maximum, using a dedicated spectrometer on a RIXS endstation. In addition to the intrinsic reduced lifetime broadening, one takes advantage of the $2 p-3 d$ repulsions in the RIXS final state that allow to disentangle XMCD signals measured with different emission energies. Nevertheless, the interpretation of CEE-MCD scans is not possible without measuring the full RIXS-MCD plane in the pre-edge range or the support of calculations.

Hence, the choice of either one or the other approach depends on the goal of the experiment. If the objective is to measure XMCD spectra with an improved resolution and sharper spectral features, the first option which consists in deconvoluting high-SNR XMCD spectra measured in Total Fluorescence Yield detection seems the most appropriate. If the goal of the experiment is to measure a $2 D$ representation in order to obtain more information on the electronic structure (such as spin orbit coupling or Coulomb repulsions acting on the $3 d$ electrons), then RIXS-MCD measurements are more appropriate. We point to the reader that both kinds of experiment require good beam stability and an additional cost in acquisition time. In addition, both XMCD and RIXS-MCD are well suited for the element selective measurement of magnetization curves.

\section{Acknowledgments}

We acknowledge ESRF for the provision of beamtime and P. Gambardella for providing the YIG single crystal. A. J. acknowledges M.-A. Arrio for fruitful discussions. M. S. acknowledges support from the National Science Centre of Poland.

\section{References}

[1] Schütz G, Wagner W, Wilhelm W, Kienle P, Zeller R, Frahm R and Materlik G 1987 Phys. Rev. Lett. 58737

[2] van der Laan G and Figueroa A I 2014 Coord. Chem. Rev. 277 95-129

[3] Carra P, Thole B, Altarelli M and Wang X 1993 Phys. Rev. Lett. 70694

[4] Thole B, Carra P, Sette F and van der Laan G 1992 Phys. Rev. Lett. 681943

[5] Mustre de Leon J, Rehr J J, Zabinsky S I and Albers R C 1991 Phys. Rev. B 44(9) 4146-4156

[6] Lu D and Rehr J J 1988 Phys. Rev. B 376126

[7] Krause M and Oliver J 1979 J. Phys. Chem. Ref. Data 8 329-338 ISSN 0047-2689

[8] Glatzel P, Weng T C, Kvashnina K, Swarbrick J, Sikora M, Gallo E, Smolentsev N and Mori R A 2013 J. Electron. Spectrosc. Relat. Phenom. 188 17-25

[9] Loeffen P, Pettifer R, Mullender S, vanVeenendaal M, Rohler J and Sivia D 1996 Phys. Rev. B 54 14877-14880

[10] Babanov Y, Ryazhkin A, Sidorenko A and Blaginina L 1998 Nucl. Instrum. Methods Phys. Res., Sect. A 405 378-381

[11] Fister T T, Seidler G T, Rehr J J, Kas J J, Elam W T, Cross J O and Nagle K P 2007 Phys. Rev. B 75

[12] Klementev K 2001 J. Phys. D: Appl. Phys. 34 2241-2247

[13] Goulon J, Goulon-Ginet C, Rogalev A, Wilhelm F, Jaouen N, Cabaret D, Joly Y, Dubois G, Corriu R J, David G et al. 2005 Eur. J. Inorg. Chem. $20052714-2726$

[14] D'Angelo P, Migliorati V, Persson I, Mancini G and Della Longa S 2014 Inorg. Chem. 53 9778-9784

[15] Filipponi A 2000 J. Phys. B: At., Mol. Opt. Phys. 33 2835-2846

[16] Goulon J, Rogalev A, Wilhelm F, Goulon-Ginet C, Carra P, Cabaret D and Brouder C 2002 Phys. Rev. Lett. 88 237401

[17] Goulon J, Rogalev A, Goulon-Ginet C, Benayoun G, Paolasini L, Brouder C, Malgrange C and Metcalf P 2000 Phys. Rev. Lett. $\mathbf{8 5} 4385$

[18] Hamalainen K, Siddons D, Hastings J and Berman L 1991 Phys. Rev. Lett. 67 2850-2853

[19] Glatzel P and Juhin A 2014 X-ray absorption and emission spectroscopy Local Structural Characterisation Inorganic Materials Series ed Bruce D, OHare D and Walton R (Wiley Online Library) pp 89-171

[20] De Groot F and Kotani A 2008 Core level spectroscopy of solids (CRC press)

[21] Carra P, Fabrizio M and Thole B 1995 Phys. Rev. Lett. 743700

[22] Hayashi H, Udagawa Y, Caliebe W A and Kao C C 2003 Chem. Phys. Lett. 371 125-130

[23] Sikora M, Juhin A, Weng T C, Sainctavit P, Detlefs C, de Groot F and Glatzel P 2010 Phys. Rev. Lett. 105

[24] Sikora M, Juhin A, Simon G, Zajac M, Biernacka K, Kapusta C, Morellon L, Ibarra M R and Glatzel P 2012 J. Appl. Phys. 111

[25] Juhin A, Lopez-Ortega A, Sikora M, Carvallo C, Estrader M, Estrade S, Peiro F, Dolors Baro M, Sainctavit P, Glatzel $\mathrm{P}$ and Nogues J 2014 Nanoscale 6 11911-11920 
XMCD with reduced intrinsic broadening

[26] Goulon J, Rogalev A, Wilhelm F, Goujon G, Brouder C, Yaresko A, Youssef J B and Indenbom M 2010 J. Magn. Magn. Mater. 322 2308-2329

[27] Castel V, Vlietstra N, van Wees B J and Youssef J B 2012 Phys. Rev. B 86(13) 134419

[28] Filipponi A, Di Cicco A and Natoli C R 1995 Phys. Rev. B 5215122

[29] Filipponi A and Di Cicco A 1995 Phys. Rev. B 5215135

[30] Cowan R D 1981 The theory of atomic structure and spectra vol 3 (Univ of California Press)

[31] Thole B, Van der Laan G, Fuggle J, Sawatzky G, Karnatak R and Esteva J M 1985 Phys. Rev. B 325107

[32] Arrio M A, Rossano S, Brouder C, Galoisy L and Calas G 2000 Europhys. Lett. 51454

[33] Gullikson E 2010 The Center for X-Ray Optics,(1995-2010), http://www. cxro. lbl. gov

[34] Juhin A, Brouder C, Arrio M A, Cabaret D, Sainctavit P, Balan E, Bordage A, Seitsonen A P, Calas G, Eeckhout S G et al. 2008 Phys. Rev. B $\mathbf{7 8} 195103$

[35] Wilke M, Farges F, Petit P E, Brown G E and Martin F 2001 Am. Mineral. 86 714-730

[36] Cabaret D, Bordage A, Juhin A, Arfaoui M and Gaudry E 2010 PCCP 12 5619-5633 